\title{
ERRATUM
}

\section{Intracortical connections are not required for oscillatory activity in the visual cortex}

\section{GEOFFREY M. GHOSE and RALPH D. FREEMAN}

Group in Vision Science, School of Optometry, University of California, Berkeley

(article appeared in Visual Neuroscience (1997), 14, 963-979)

Due to a software translation incompatibility, errors were introduced in the final version of the manuscript submitted for the above publication. Readers are advised to note the following corrections:

On p. 965 , the first line of text below equation (1) should read "where $m$ is the order of the gamma function, $\lambda$ is the coefficient of variation ...."

On p. 965, the last line of text above equation (3) should read "the assumption of equal weights among the inputs $\left(w_{i}=1\right.$ for all $\left.i\right)$ :"

On p. 971, equation (7) should read

$$
E(t>0)=\left[\left(1-e^{-\gamma t}\right)-C\right] \theta
$$

On p. 971, the last sentence of the second full paragraph in the right hand column should read "The simulated complex cell has a firing threshold $\theta$ of 4.5 [eqn. (3)] and is discharged at a rate of 82.5 spikes/s when stimulated and 0.3 spikes $/ \mathrm{s}$ in the absence of stimulation."

On p. 972, in the third and fourth lines of the first column the $q_{I}$ should be replaced by $\theta_{l}$.

On p. 972, in the twelfth and thirteenth lines of the first column the " $g$ " should be replaced by a " $\gamma$ " for the decay constant.

On p. 974, the twenty-first line of the first column should be changed to read "However, in the visual cortex, field potentials are largely generated by ...."

A corrected version of the paper is reproduced on $\mathrm{pp} .963 \mathrm{R}-979 \mathrm{R}$ following. 